\title{
Evaluation of work Posture by RULA and REBA: A Case Study
}

\author{
N. A. Ansari, Dr. M. J. Sheikh \\ Mechanical Department Agnihotri college of Engineering Wardha 442001, India \\ Mechanical Department Bapurao Deshmukh college of EngineeringSewagram, India
}

\begin{abstract}
The common occupational problem of the workers is musculoskeletal disorders in India. Currently the work is being carried out manually in most of the small scale industries therefore the issues of work related musculoskeletal disorders and injury in different body sites are of top priority. Postural analysis tool using Rapid upper limb assessment (RULA) and Rapid entire body assessment (REBA) were applied for assessment which indicates that the workers are working above the secure limit. This ergonomic study sheds light on posture analysis of the workers in small scale industry. The study was conducted on 15 workers engaged in small scale industry situated at MIDC Wardha (Maharashtra, India). Video tape on different activities of the workers was prepared and then images were cropped from it for the analysis. This study presents assessment of work posture of workers engaged in different activities of small scale industry. Evaluation of posture was carried out using RULA and REBA. Assessment is carried out using worksheet. The RULA method determined that the majority of the workers were under high risk levels and required immediate change. The REBA method determined that some of the workers were under lower levels and majority at high risk levels. Hence it was concluded that; there is a lack of ergonomics awareness and understanding in small scale industries. Evaluation using postural analysis by RULA and REBA indicates that the workers are working above the secure limit. The major percentage of the workers having awkward postures. Thus the workers are under moderate to high risk of Musculoskeletal disorders.
\end{abstract}

Keywords: Musculoskeletal disorders (MSDs), RULA, REBA, SSIs, ergonomics.

\section{Introduction}

Small scale industry plays a vital role in development in countries like India; they play an important role in employing the majority of the industrial workers. The majority of workers are suffering from musculoskeletal disorders which is a most common work-related problem in India. In small scale industries most of the work is still carried out standing and manually hence issues of work related musculoskeletal disorders and injuries in different parts of the body are of great concern. RULA (rapid upper limb assessment) is a survey method developed for use in ergonomics investigations of workplaces where work-related upper limb disorders are reported [1]. The REBA is a postural analysis tool sensitive to musculoskeletal risks in a variety of tasks and assessment of working postures found in health care and other service industries [2].Performing jobs in prolonged standing has contributed numerous health effects such as work-related musculoskeletal disorders, chronic venous insufficiency, preterm birth and spontaneous abortion, and carotid atherosclerosis. However, those injuries can be minimized through application of engineering and administrative controls [3]. Lack of knowledge about ergonomics is observed in industry in which work is carried out. Musculoskeletal disorders are there in the welding process where workers are working in kneeling posture and it shows that there is need to change the body postures [4]. The application of ergonomic principles would help to increase machine performance and productivity, but mostly help human operator to be comfortable and secure [5]. Workers work under tough conditions to perform the desired task. These tough conditions normally give rise to various musculoskeletal disorders within the workers. These disorders emerge within the workers body due to repetitive lifting, differential lifting height, ambient conditions etc [6]. The importance of ergonomics in product design, working environment, and its influence in industrial workstation design, the interdisciplinary nature of ergonomics and the implications of ergonomics in industrial engineering function [7]. The study revealed that there had been several gaps in work environment, tools and equipment that affect the health and safety of workers at the work site [8]. Ergonomics related to the design of methods and processes can help eliminate or decrease work related risks as well as improve the company's quality and productivity [9]. Awkward posture, lifting, forceful movement and manual work at rapid rate contribute to musculoskeletal disorder. Present study is focused on assessing the work posture of worker engaged in different activities of casting [10]. The application of ergonomic principles would help to increase machine performance and productivity, but mostly help human operator to be comfortable and secure [11]. It was concluded that: there is a lack of ergonomics planning and methods in small scale forging industry. A significant proportion of the workers are working in very bad postures. The study recommended proposed that there is dire need of implementation of ergonomics intervention with proper awareness among worker [12]. Study concluded that an ergonomic workstation design 
can contribute significantly to improve physiological performance of the operators [13]. Overall result shows that the frequency and level of discomfort occurrence was at lower risk to all the body parts. Even though the practice of combination of static and dynamic standing working system, and by varying leg position while standing assist in reducing discomfort from working in standing position, that did not apply to all standing operators [14].The aim of this work consists in developing a design methodology for preventive ergonomics and comfort analyses of Human-Machine-Interface (HMI) [15].

\section{Methods And Material}

This research study is conducted at small scale industry situated at MIDC Wardha (Maharashtra). The 15 workers were selected for study of average stature $168.34 \mathrm{~cm} \pm 2.69$ S.D., average age 35.8 years \pm 3.02 S.D., average weight 63.6 kilograms \pm 6.66 S.D. and average experience 11.2 years. The video recording of their postures showing movements of the workers during working was recorded. After footage the video, it was cropped to get snapshots for the analysis of posture of the worker. Snapshots of 15 workers performing their work were obtained. The snapshots were analyzed to fill the scores in RULA and REBA; score sheets (appendix). The first step was overall body posture assessment using REBA method. The works with the involvement of high risk were scored higher and those with less risk involvement were scored 0 . Immediate corrective actions and necessary changes were recommended for activities scored higher to keep away from any risk.

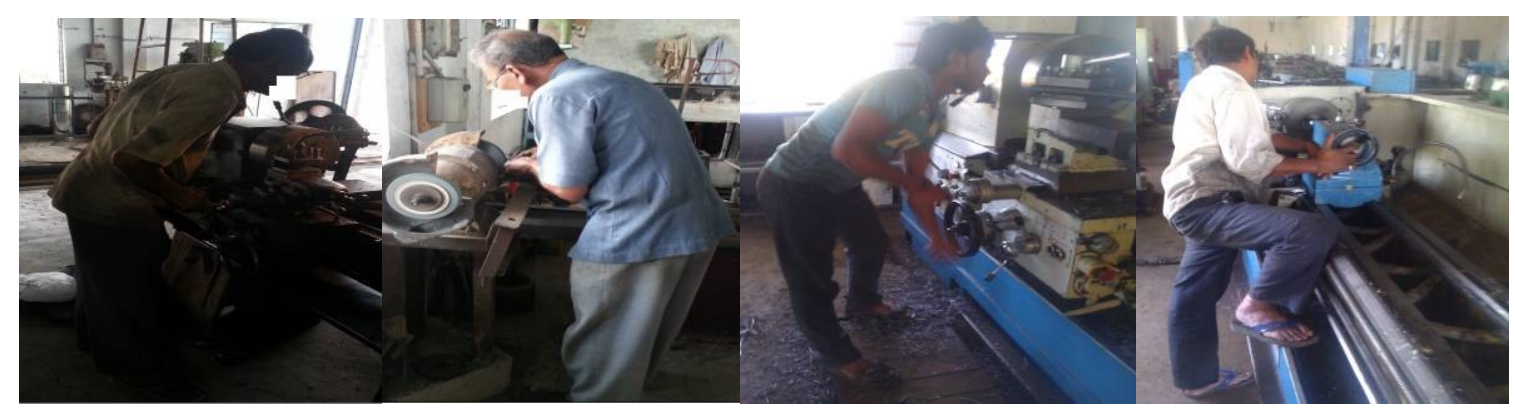

FIGURE 1: Postures adopted during working.

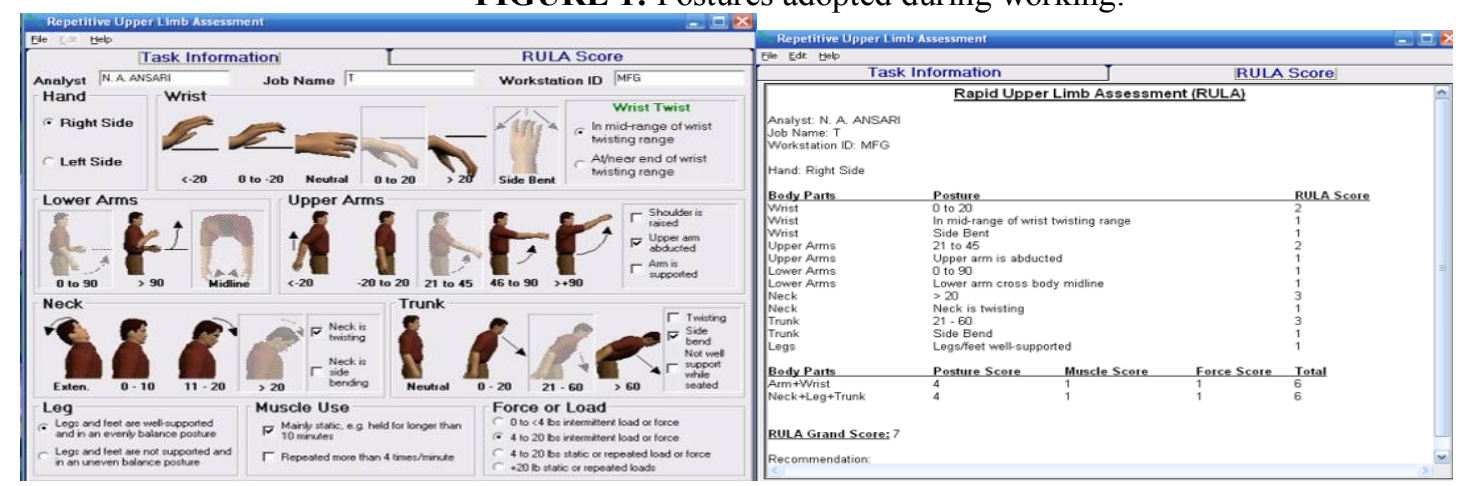

FIGURE 2: Assignment of RULA Score according to position of body part (Source: Ergointelligence ${ }^{\mathrm{TM}}$ Software.)

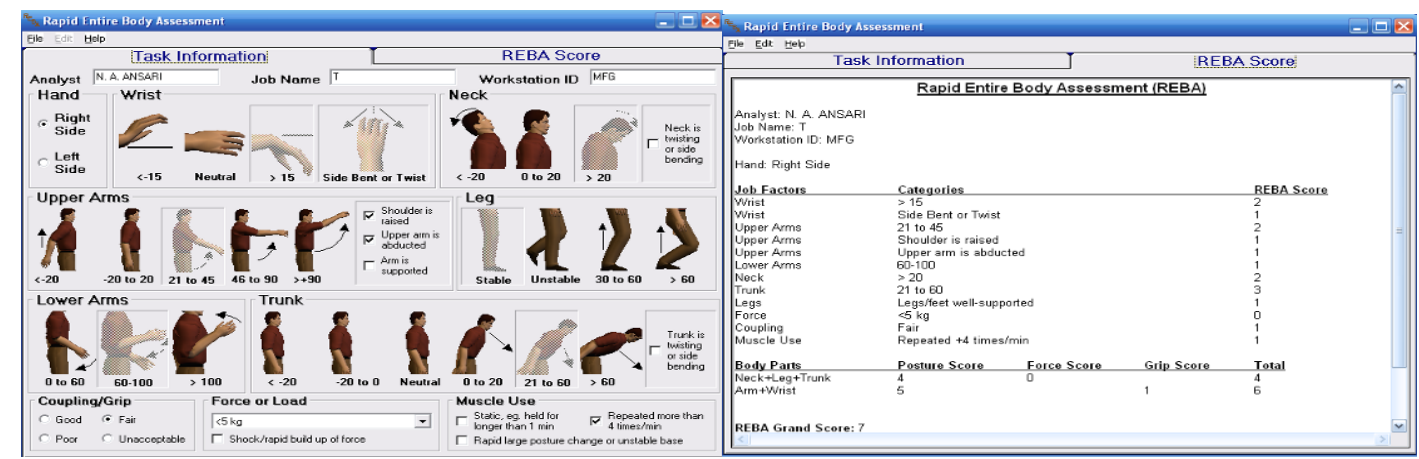

FIGURE 3: Assignment of REBA Score according to position of body part (Source: Ergointelligence ${ }^{\mathrm{TM}}$ Software.) 
The selected photos of workers performing their work at the manufacturing unit are shown in Figure 1. The critical analysis of the observational data and recorded videos has been carried out. The RULA and REBA Score were calculated using Ergointelligence ${ }^{\mathrm{TM}}$ software and as an illustration the calculation for posture 1 is presented. Ergointelligence ${ }^{\mathrm{TM}}$ software was also utilized to verify the results; the input and output screen shots of the same are shown in figure 2 and 3 respectively. Then ergonomic risk factors were identified by analyzes the results using this application.

The upper limbs mainly arms and wrist of posture was assessed using RULA score sheet; the range of movement for each body site is divided into sections. These sections are scored so that the score 1 is given to the range of movement or working posture where the risk factors present are minimum. Higher scores are allocated to sites of the movement range with more extreme postures indicating an increasing presence of risk factors causing load on the structures of the body segment. The exposure scores according to RULA were divided into four $0,1,2$, and 3 exposure categories: negligible, low, medium and high respectively. Medium and high risk actions should be urgently addressed to reduce the level of exposure of risk factors. For those activities where whole body and limbs motion needs to be assessed REBA was used which is also a pen paper technique. In REBA the body parts are divided into sections and each body part is scored according to its range of movement. Higher scores are given to the body parts where presence of risk factors are more and lower scores are given to those where presence of risk factors are minimum. The REBA scores were divided into five $0,1,2,3$, and 4 are categories: negligible, low, medium, high and very high. Medium, high and very high needed an immediate action to keep away from any musculoskeletal disorder.

\section{Results And Discussion}

The assessment using RULA worksheet is presented in appendix. Table 1 presents the different categories of the risk levels as obtained after analyzing the posture.

\begin{tabular}{|l|c|c|c|c|}
\hline RULA Level & 0 & 1 & 2 & 3 \\
\hline RULA score & $1-2$ & $3-4$ & $5-6$ & 7 \\
\hline Risk Level & Negligible & Low & Medium & High \\
\hline Reqd. Action & Acceptable & $\begin{array}{c}\text { Investigate } \\
\text { further }\end{array}$ & $\begin{array}{c}\text { Investigate further and } \\
\text { change soon }\end{array}$ & Investigate and change immediately \\
\hline $\begin{array}{l}\text { Percentage of } \\
\text { workers }\end{array}$ & -- & 13 & 47 & 40 \\
\hline
\end{tabular}

TABLE 1: Categorization under RULA Level

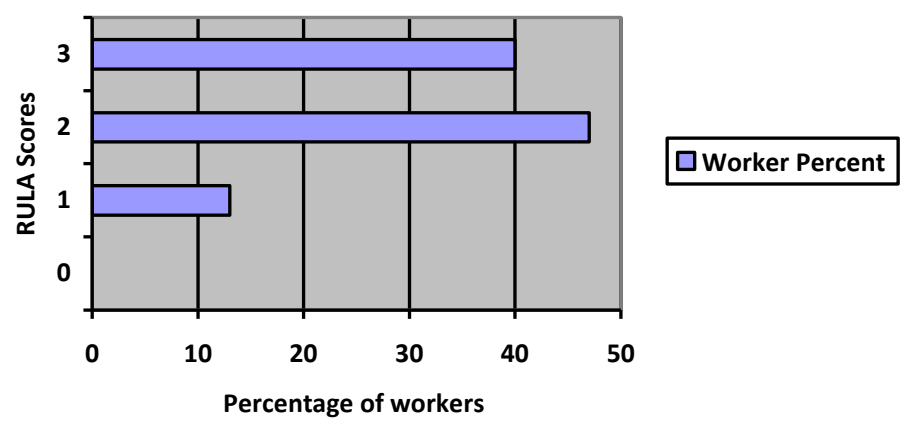

FIGURE 4: Percentage of worker under RULA Level

Figure 4 showed that around $40 \%$ of the workers are at high risk level and needs a Investigate and change immediately, whereas $47 \%$ workers were found at medium risk levels and needs a Investigate further and change soon. Around $13 \%$ of the workers are working in Investigate further. The results of the posture analysis using RULA are shown in Table 1 . These results reveal that all categories of the risk levels exist in jobs postures. The table showed that posture of $40 \%$ workers who used in performing the activities is at high risk levels. A further investigation with an immediate change was recommended to these workers. The table also shows that none of the worker is at negligible risk level. The study was done on workers working in different sections of the industry and their activities were divided in different categories. The posture analysis was done according to these activities using the same sequence of RULA and REBA. 
Using the REBA analysis method, it was observed that the major parts of the workers were working in unacceptable posture at high risk levels.

\begin{tabular}{|c|c|c|c|c|c|}
\hline REBA Level & 0 & 1 & 2 & 3 & 4 \\
\hline REBA score & 1 & $2-3$ & $4-7$ & $8-10$ & $11-15$ \\
\hline Risk Level & Negligible & Low & Medium & High & Very high \\
\hline Reqd. Action & None necessary & May be necessary & Necessary & Necessary soon & Necessary now \\
\hline $\begin{array}{c}\text { Percentage of } \\
\text { workers }\end{array}$ & -- & 13 & 33 & 53 & - \\
\hline
\end{tabular}

TABLE 2: Categorization under REBA Level

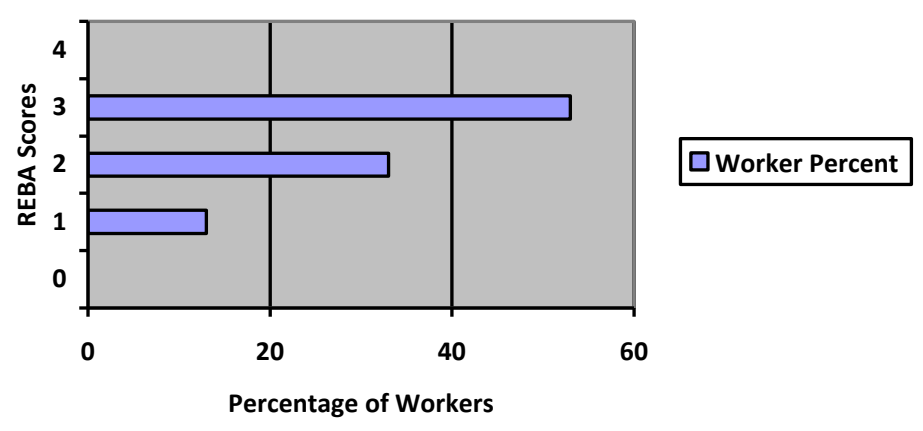

Figure 5: Percentage of worker under REBA Level

Around $53 \%$ of the workers were working at high risk levels. It was found that, if the workers continued to work in the same posture they suffer from the MSDs related to neck, trunk and wrist in the near future. It was recommended to take the corrective action as soon as possible. However, when these units were studied using the REBA tool, REBA showed that most of the workers in the drilling operation were working in acceptable posture and a necessary change may be required for them. Around 53\% of the workers were working at high risk levels and their neck, trunk and wrist were under high physical strain and needed a necessary action soon. Around $33 \%$ of the workers in the jobs were at medium risk levels and needed a necessary change. Some of the workers in the turning jobs were bending their trunk to unacceptable limit and most of them had upper arm under high strain. The workers were suggested to keep their trunk straight while working. Also, in some jobs the workers were bending their trunk to a higher degree which was not acceptable and they needed an necessary change. However, in rest of the activities the workers were working in fine posture and they may require a necessary action.

\section{Conclusion}

The evaluation of body posture has been carried out for this particular manufacturing unit by RULA and REBA tools, it can be concluded that; significant proportion of the workers are working in uncomfortable and painful postures as found by analysis. This is due to lack of ergonomics knowledge and awareness in small scale industry. Thus the workers are under moderate to high risk of musculoskeletal disorders as determined from REBA and RULA risk level. This study recommends the immediate implementation of ergonomics interventions with proper knowledge among workers and health education on common postural change, implementation and monitoring of laws among industries are recommended to take down morbidity due to musculoskeletal disorders. 


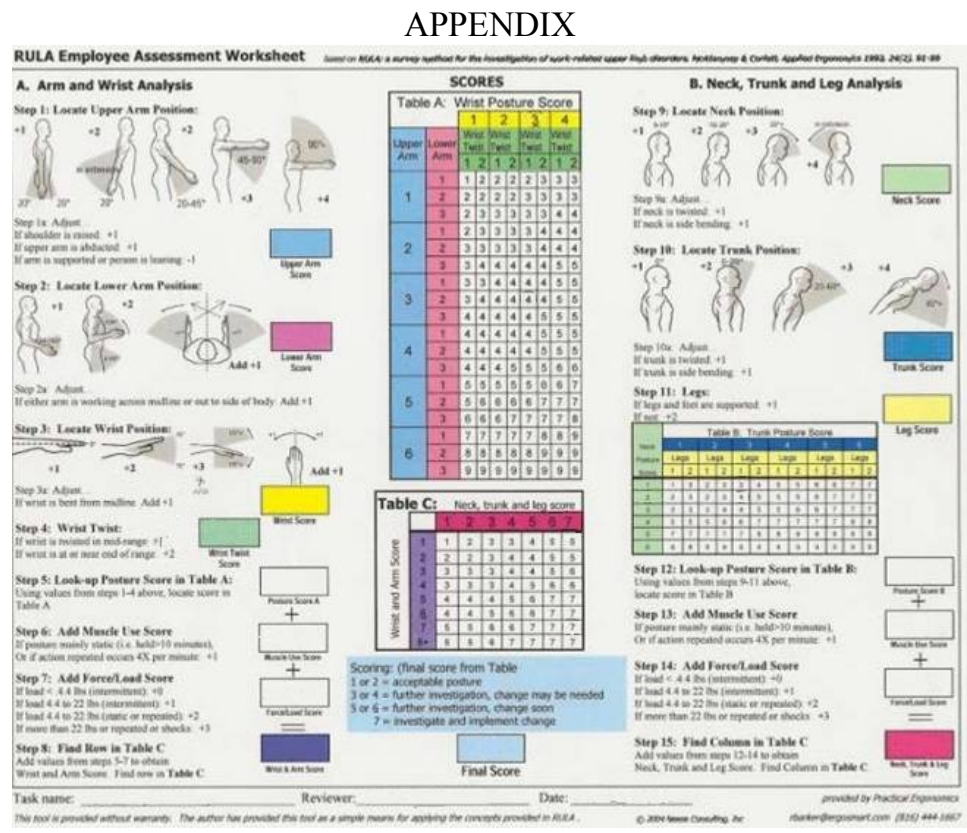

Source : McAtamney, L. \& Corlett, E.N. (1993)

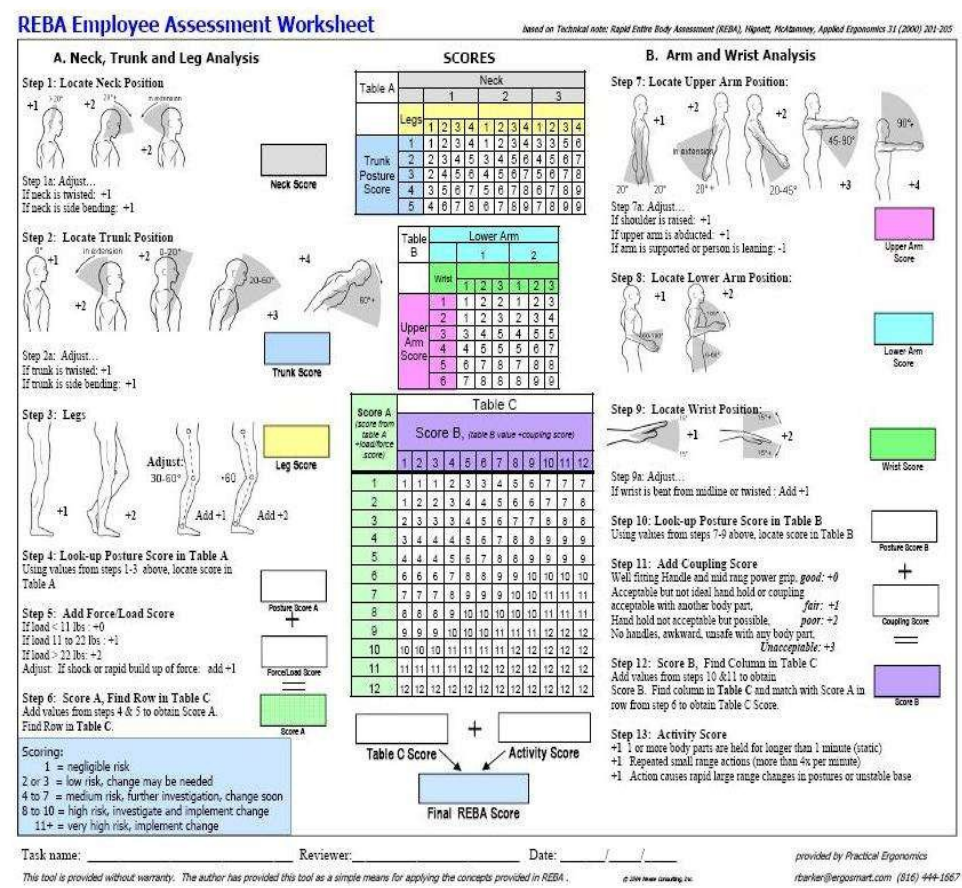

Source : HIGNETT S AND McAtamney, L. (2000)

$+$

\section{References}

[1] Lynn McAtamney et al., "RULA: a survey method for the investigation of world-related upper limb Disorders," Applied Ergonomics, 1993

[2] Hignett, S. and McAtamney, L., "REBA: A Survey Method for the Investigation of Work-Related Upper Limb Disorders," Applied Ergonomics, 2000

[3] Isa Halim \& Abdul Rahman Omar, "A Review on Health Effects Associated with Prolonged Standing in The Industrial Workplaces," International Journal Of Research And Review In Applied Science, vol. 8, issue 1, July 2011

[4] D. N. Agrawal et al.," Study and Validation of Body Postures Of Workers Working In Small Scale Industry through Rula," International Journal of Engineering Science and Technology, Vol. 3 No.10 October 2011

[5] Aide Maldonado-Macias et al., "Ergonomic Evaluation of Work Stations Related With the Operation of Advanced Manufacturing Technology Equipment: Two Cases of Study", XV CONGRESO INTERNACIONAL DE ERGONOMIA SEMAC, 2009

[6] Aman Sachdeva et al., "Minimizing Musculoskeletal Disorders in Lathe Machine Workers," International Journal of Ergonomics, Volume 1 Issue 22011

[7] Mohammad Iqbal et al., "Ergonomics and Design," International Conference on Industrial Engineering and Operations Management Kuala Lumpur, Malaysia, January $22-24,2011$ 
[8] Parimalam P. et al., "Ergonomic interventions to improve work environment in garment manufacturing units," Indian Journal of Occupational and Environmental Medicine, August 2006 -Volume 10 - Issue 2

[9] M. C.I. Javier Antonio Lom Holguín et al., "Ergonomics and its relationship to the design of production processes," PonenciaConergo2008

[10] Lakhwinder Pal singh., “An Investigation Work Posture Of Workers Engaged In Casting Industry: A Study In India,”Asian Journal of Managerial Science, Volume 1 Number 1, January - June 2012

[11] Asim Zaheer et al., "Ergonomics: A work place realities in Pakistan," International Posture Journal of Science \& Technology, Volume 2, Issue 1, April, 2012

[12] L. P. Singh., "Work Posture Assessment in Forging Industry: An Exploratory Study in INDIA," International Journal of Advanced Engineering Technology, vol. 1 issue III Oct.-Dec. 2010.

[13] Isa Halim et al., "Posture, muscle activity and oxygen consumption Evaluations among metal stamping operators: A pilot study in Malaysian small and medium Industries," Journal - The Institution of Engineers, Malaysia, Vol. 72, No. 4, December 2011.

[14] Zahari Taha et al., "Frequency and Level of Discomfort of Male Operators in Standing Work Posture," The 9th Asia Pasific Industrial Engineering \& Management Systems Conference. APIEMS 2008

[15] A Naddeo et al., "Postural analysis in HMI design: an extension of OCRA standard to evaluate discomfort level," Journal of Achievements in Materials and Manufacturing Engineering, Vol 39 Issue 1 March 2010 